\title{
Crafting Soft Geometry Form and Materials InForming Analog and Digital Craft Processes
}

\author{
> Jonathon Anderson \\ University of Nevada Las Vegas, USA \\ Jonathon.anderson@unlv.edu
}

\author{
$>\quad$ Ming Tang \\ University of Cincinnati, USA \\ tangmg@ucmail.uc.edu
}

\begin{abstract}
This paper outlines a methodology that adds to current craft-based discourse in the digital age. It proposes pedagogy centered on a constant examining of the parametric relationships between form, material, and load. The paper illustrates how materials and loads were integrated as datasets into "soft geometry" modeling and installation pipelines that further explore a hybrid process that incorporates materials and craftsmanship. The results expand the boundary of conventional static form and spatial interaction within the deformation rules (material and force) while seeking form through the exploration of both digital simulation and analog techniques.
\end{abstract}

Keywords: digital-physical; craft; soft geometry; form; material

\section{Introduction}

Digital fabrication and modeling has, for better or worse, radically changed the way we perceive and engage craft. Our contention is that digital technologies allow designers to prototype, to the highest precision, but is ultimately still viewed as a final result or representation of a digital model. Digital fabrication is no longer about the process of craft itself. With these ideas in mind, we began to ask questions like: Has the machine made us all tradesman? Ho do we marry old boundaries with advanced technology? How do we teach craftsmanship in a digital environment?

Like many others, we define craft as a "process of careful and deliberate planning, skill, and execution where a consistent negotiation happens between artist and the object" (Oxman, 2007). In this process, the object and tools are not clearly defined but rather inform a gradual form finding process. Pye's concept of workmanship where "the quality of the result is not predetermined, but depends on the judgment, dexterity and care which the maker exercises as he works" (Pye, 1968) aligns our pedagogy with a non-determinate use of technology. In this paper, two student projects, developed at two universities, are presented through the lens of learning and gaining a deep appreciation for craft and material. Additionally, simulated deformation of elastic material and post-installation analysis were explored to better understand what influenced design decisions around form, materials, and craft instead of thinking about the rigidity or stiffness of the form. This hybrid design process teaches students how the act of creation is influenced by a constant negotiation of load, material and form.

\section{Methods and Materials}

McCullough describes craft as "the application of personal knowledge to the giving of form" (McCullough, 1996) and yet today it seems the majority of "digital craftsman" are loosing touch with material and their relationships to tools. Instead, these important attributes were replaced with post processing, a final execution, and an unthinkable precision. In an act of craft there is a moment of being uncertain and under constant evaluation or negotiation, so how is it that we define these "experimental" parameters to students and explain that machines precision does not always imply craft. We recognize the amount of discourse around craft and automation, but we ask how this discourse redefines our notion of craft and the processes that emerge or parallel our traditional understandings. A marriage of hand and automation will perhaps move closer to our answer or as Joseph Choma believes "a hybrid fabrication process which combines digital fabrication with hand craft techniques... a slightly slower process which yields a sensibility to intimacy" (Choma, 2010).

Through collaborative teaching and research, the authors have explored this idea using two reversed workflows; one being digital to simulation to physical, and the second being physical to digital to simulation. In both cases students understood the idea of craft being centered on the discovery, constant struggles, meticulous attention to detail, and the idea that final output was not going to be crafted solely by a machine.

The research methodologies utilized in this study are arts-based, heuristic, and qualitative. The qualitative method of observation 
provided data regarding the development and realization of the project during the act of making in the form of field notes, process photos, and videos. Data was analyzed in terms of precision, subtractive techniques, sequences of actions, usage of time, team interaction, ability to utilize an iterative process, and material understandings. This research documented the "translation of drawings into computer code" (Lynn, 2008) while understanding how the material itself influenced the process or decisions. Arts-based research methods were used in the production of building of study models, material testing, and case studies as a way to compare the digitally designed and material influence of the actual realization of the designs at full scale. Heuristic methods such as trial and error and intuitive judgment were used in order to learn about programming, discover efficient coding techniques, material factors, craft, and solve problems based on the researcher's previous knowledge (Sela-Smith, 2002) in digital fabrication and design. Through this craft based creative research, interdisciplinary relationships among craft, art, design, computer science, and computational means were examined in order to expand the notion of craft in both digital and analogue processes, improve current craft techniques, optimize design process with material inputs, and generate a closed loop of information between human, robotic, and material systems.

\section{Results}

The two-presented case studies were collaborative efforts at two universities but under the direction of the authors. Our interest and research in this topic, paired with the development of two projects, allows us to critically evaluate the process, impact, and student development. The interest of authors' lies in rethinking and questioning the ways in which we are teaching craftsmanship through hybrid digital and analog processes.

\section{Cumulus Oculi: digital to simulation to physical}

Cumulus Oculi, a speculative studio project, is conceived as a built environment personified through light. The installation made from fourteen apertures; half of the apertures contain a sensor, and half of the apertures contain light sources. The installation is dormant in its solitary state and awakened by the user who navigates through the space while triggering the seven sensors. The sensors located within specific apertures detect user's movement through space and light illuminates inside a corresponding aperture once a user triggers a sensor. The user experiences the environment's awakened state through its responsive illumination and eventually returns to dormancy. The environment's unpredicted response causes users to analyze how their interactions affect the environment and map peoples interactions and movements through space.

The project was digitally designed with the intention of investigating soft surfaces through laser cutting and tabbing paper components. Strict conditions were held in the computer and the laser cutting of some 25,000+ unique triangles; it was not until the installation that the students began to take on the attributes of a craftsman and realize the freedom of paper as a material (figure 1). A computer simulation model was calculated to account for basic sag and displacement of the paper installation based on its
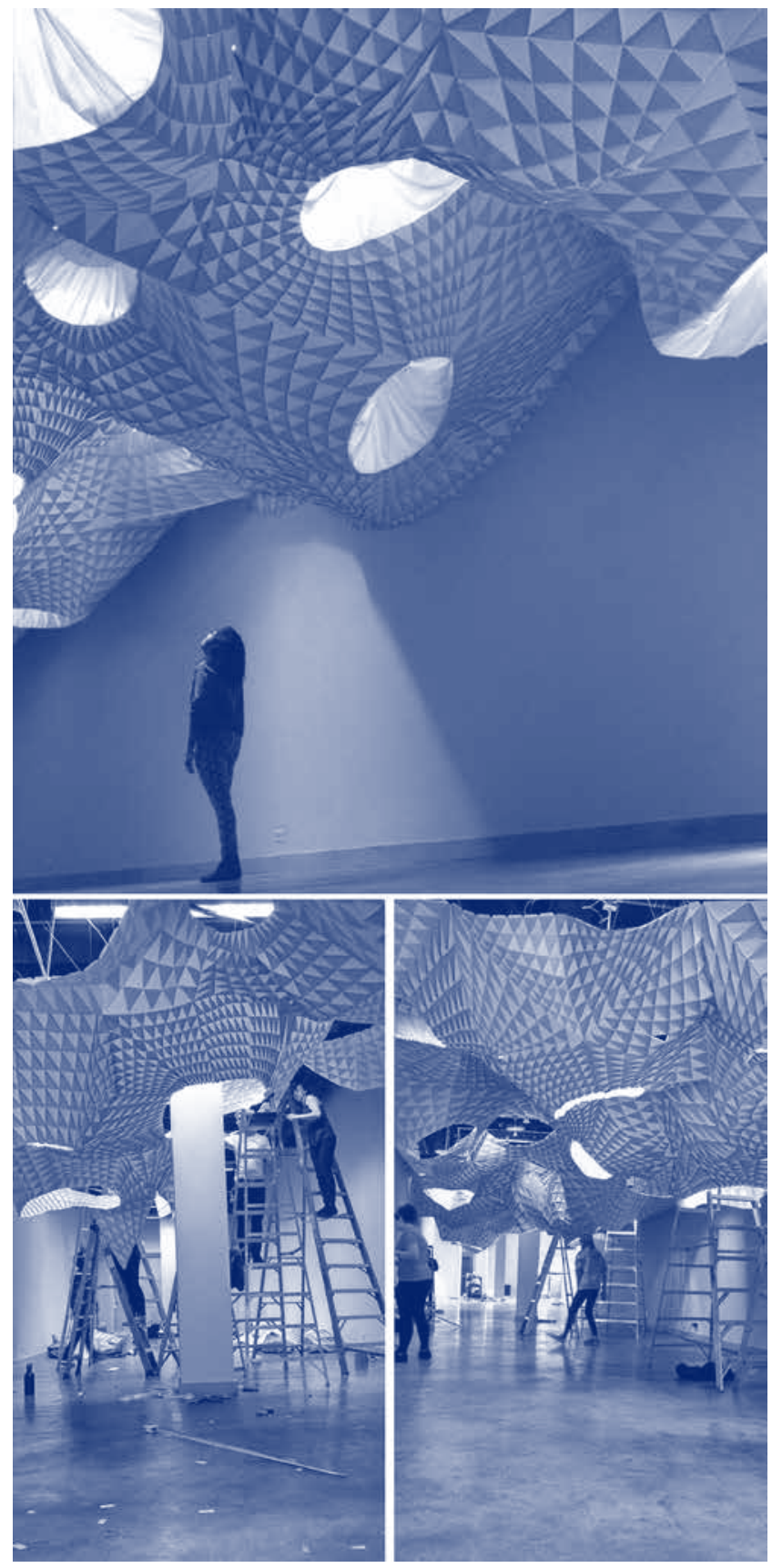

Figure 1: top: completed installation bottom: installation process.

load. However, there are other factors that couldn't be statistically determined such as the demonstrated craft in connecting faces or wear and stress on the material over extended periods of time and handling (figure 2). The final deformation of an elastic surface was the result of various dynamic forces simultaneously applied to the material.

Cumulus Oculi was designed and conceived in seven weeks in a vertical studio comprised of five juniors and four senior interior architecture students. Students involved in this process were responsible for developing the concept, digital models, exploration models, prototypes, grashopper definitions, and the 

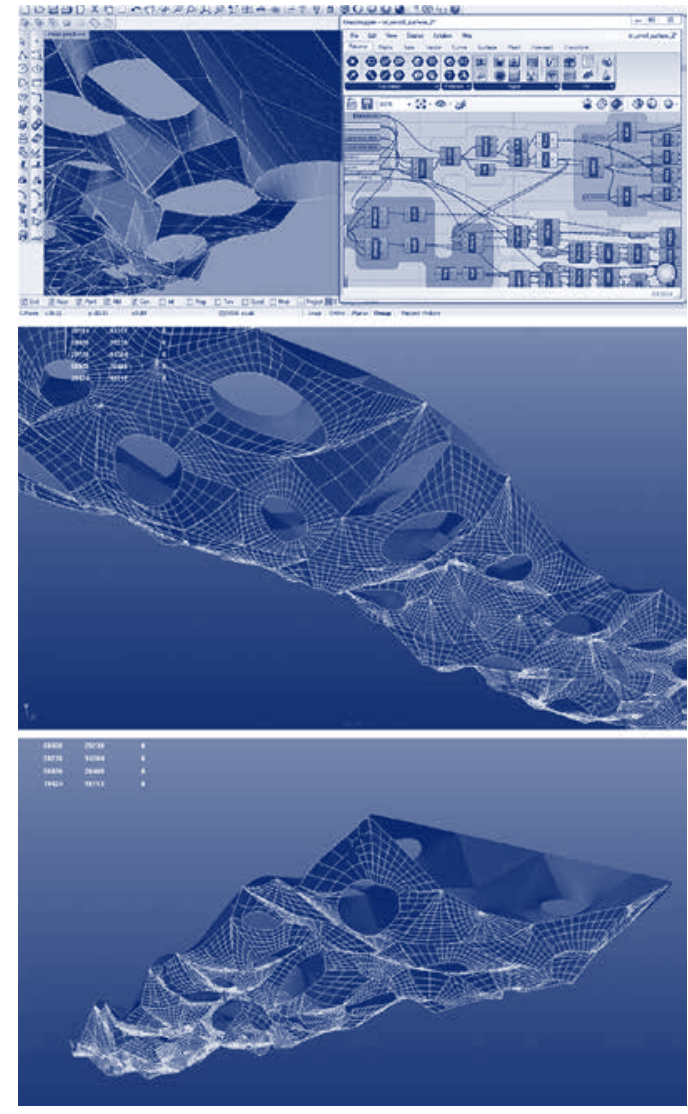

Figure 2: digital construction of stiff surface. Middle + bottom - red surface shows computer model where blue surface shows sag and deformation according to material stiffness. The new surface adapted the load by changing its shape. A triangulated mesh was modeled in Rhino to match the physical installation, then a simulation model was constructed in Autodesk Maya by assigning stiffness values and constrains to a soft geometry.

actual production of the final installation. The instructor assisted the students in various processes along the way but mostly worked on the logistics of installing the design within a local gallery. A natural hierarchy emerged in the studio with more experienced computer users developing the computational means while handson students explored the material itself and the influence it would have on the design decisions. All students fabricated and aggregated the final installation once the design was finalized and optimized based on craft and material inputs. The apertures were assembled off site as individual components that could be strung into place and connected to adjacent oculi. For obvious reason to us, the students experienced a great deal of difficulties in installation what they conceived a highly refined digital design.

Digital Catenary: physical to digital to simulation

In this project, students used a reversed design process, compared to the Cumulus Oculi project. The physical rule became the design driver to generate the entire steel frame and tensile structure. Digital Catenary studied the implementation of parametric thinking in the creation of a soft geometry that is an adaptable and scalable system responding to material itself and external forces. A cable structure was introduced to demonstrate its ability to adapt to changing loads by changing its own shape. The relationships between the cable shapes to multiple concentrated loads were studied using physical prototypes. Students later used a digital simulation to compare the results. For example, a simulated concentrated load made the cable forms two straight lines meeting the points of load application. A uniformly distributed vertical load across the span will make the cables form a parabola. A catenary form was achieved by simulating the load distribution along the length of cable.

Students were tasked with understanding and developing a 3D form that capitalizes on the fluid nature of cables. The mathematic relationship between load, tension, span and sag were investigated, as well as general knowledge about how to anchor cables at their ends to prevent movement caused by lateral and uplifting loads. In a digital simulation modeled in Autodesk Maya, Gaudi's catenary model was analyzed and informed students' structural and formal investigations of tensile structures and developed connections between form, various constraints, materials, and different forces. The objective was to decode the analog process once used by Gaudi and recreate it as a digital simulation. In the digital simulation, variables such as air dynamics, material properties, and forces integrated through Maya's Nucleus physics engine, which generated both threedimensional form, time-based media to visualize the optimization process, structural performance and material calculations. Students set up different physics rules, material properties, and collision objects to investigate how the fabric form and tensile structure can negotiate with these conditions and to compare digital results with the analogue model. For example, students used the kinetic frames to deform the attached tensile structure, or used simulation engine to generate the hyperbolic surface rather than direct creates the forms based on already known math formulas.

Students build several physical prototypes before they build the digital simulation (Figure 3). In the physical model, students were encouraged to build layers of stabilizing cable to stiffen the primary cables, including inner ring, outer ring, and radial cable system. The stability of the prototype is evaluated by blowing wind with a hair dryer. Then the prototypes were reconstructed in a digital simulation. The physical deformations were compared with the result in Maya's wind tunnel simulation.

After evaluating early research, students at the University of Cincinnati were challenged with the 2013 ACSA Fabric in Architecture competition. The development of these projects was to take place in an architecture studio. The building program for this competition was a medium size hotel with tent and membranes structure in Cincinnati, Ohio. Knowledge gained in early research was successfully transferred to a complex tension membrane that stretched over a hyperbolic steel frame. Students focused on case studies of Omar and Frei Otto's precedent, as well as the latest synthetic fabrics technology. Students used a reversed design process, compared to the Cumulus Oculi project where physical rules became the design driver to generate the entire steel frame and tensile structure. Three essential design elements were emphasized, including the parametric relationship between steel 


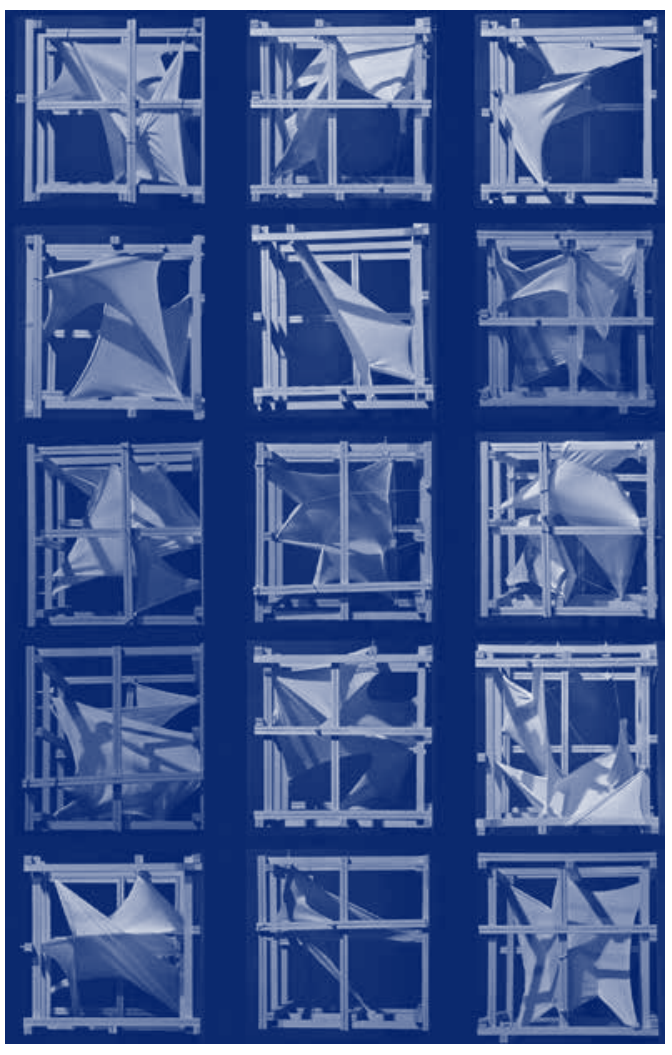

Figure 3: physic based form seeking using cables and fabric.

frame and the organic fabric (material driven), the simulated load and stability study, as well as of sun shading optimization based on environmental factors. One of the student projects at the University of Cincinnati, designed by student Trey Meyer, won the first place of the competition (Figure 4).

\section{Discussion}

The presented projects examine approaches where materials and load were set and integrated into the digital modeling and installation pipeline. This hybrid process explores an approach that incorporates materials and craftsmanship into the early stages of design decisions. The results expand the boundary of conventional form seeking through material exploration by extending the spatial interaction within the deformation rules (material and force) and their controlled objects while seeking form through digital and analog techniques. Simulated deformation and post-installation analysis has also influenced designers to think of form, materials, and craft. Here, the formal order of deformable components is decentralized from the predetermined form and exclusively ordered through its relation with all other elements within the system. Instead of thinking about the form as the rigid geometry, this hybrid design process has taught students to specify the process of creation through a constant negation of load, material and form.

The use of soft geometry runs contrary to our architectural intuition of tectonics and material. Instead of rigid or static form, the research presented within the paper looks to expand on the

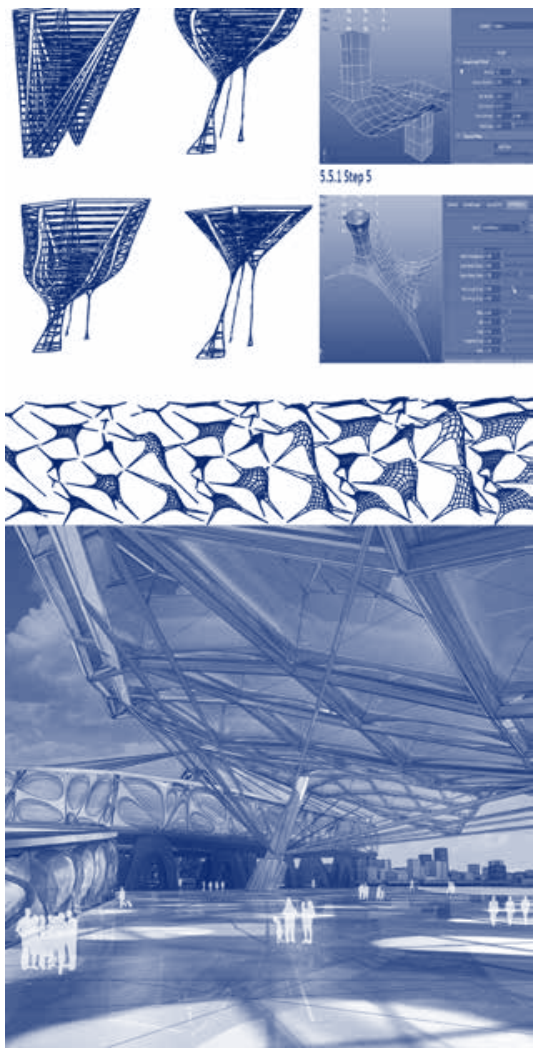

Figure 4: Top: rules of physics. Parametric design using simulated dynamic forces and fabric properties. Bottom: Winning entry of student Trey Meyer, University of Cincinnati.

nature of softness, morphing and adaptable form that provides another perspective to view architectural design as a movement, changing shapes adapted to various structural, environmental and material parameters. Students understand how craft develops through constant negotiation and integrating hybrid processes into their chosen workflow. The new equilibrium composed by these changing parameters and variables will allow the soft geometry to become eventually stable under a balanced condition.

\section{References}

Choma, J. (2010) Contested Boundaries: Digital Fabrication + Hand Craft. Sigradi Conference Proceedings.

Lynn, G. (2008). Greg Lynn Form, New York: Rizzoli.

McCullough, M. (1996) Abstracting Craft: The Practiced Digital Hand. MIT Press: Cambridge, MA.

Oxman, N. (2007). Digital Craft: Fabrication Based Design in the Age of Digital Production, in Workshop Proceedings for Ubicomp 2007: International Conference on Ubiquitous Computing. September; Innsbruck, Austria; 534-538

Pye, D. (1968) The Nature and Art of Workmanship. Cambridge University Press: Cambridge, MA.

Sela-Smith, S. (2002). Heuristic Research: A Review and Critique of Moustakas's Method. Journal of Humanistic Psychology, 42(3), 53-88. 